\title{
Evaluation of the link between pelvimetry based on computed tomography and predicting status' delivery
}

\author{
Shahla Mirgalobayat, ${ }^{a}$ Laya Ghahari, ${ }^{b}$ Leila Allahqoli, ${ }^{a}$ Seyed Reza Saadat Mostafavi, ${ }^{c}$ Katayoun Safari,
}

Masih Rikhtehgar, ${ }^{c}$ Ali RamezanGhorbani, ${ }^{c}$ Mohmoodreza Madadiane

\author{
aEndometriosis Research Center, Rasoul-e-AkramHospital, Iran University of Medical Science (IUMS), Tehran, Iran \\ bDepartment of Anatomy, School of Medicine, Aja University of Medical Sciences, Tehran, Iran \\ 'Department of Radiology, Rasoul-e-Akram Hospital, Iran University of Medical Sciences, Tehran, Iran \\ dDepartment of Small Animal Internal Medicine,Islamic Azad University, Tehran, Iran \\ eFaculty of Pharmacy, Islamic Azad University, Tehran, Iran \\ Corresponding author: Shahla Mirgalobayat (Email: mirgaloybayat.sh@iums.ac.ir) \\ (Submitted: 14 August 2019 - Revised version received: 02 September 2019 - Accepted: 12 September 2019 - Published online: 26 December 2019)
}

Objectives The aim of this study is an anthropometric study in non-pregnant reproductive-aged women to predicting kind of delivery.

Methods This study was based on the archived information of Hazrat-e Rasool university hospital on 157 generative age women from 16 to 60 years old who underwent CT between March 2015 and March 2018.

Results The obtained results showed that the three conjugates on sagittal plane, transverse diameter, anteroposterior sagittal diameter, interspinous diameter, and intertuberous diameter on the coronal plane. The obstetrical conjugate was $123.3 \mathrm{~mm}$ in normal vaginal delivery and $113.9 \mathrm{~mm}$ in Cesarean section, which was slightly longer than delivery group. The mean interspinous and intertuberous diameters measured by CT scans were 105.0 and $107.4 \mathrm{~mm}$.

Conclusion The results of our study showed that because of the reduction in diameter of the inlet and middle pelvis, the rate of cesarean section was higher in females under 35 years ages .

Keywords pelvimetry, computed tomography, non-pregnant women

\section{Introduction}

The pelvimetry refers to the evaluation of the diameters of the bony pelvis to assess the pelvic cavity for the passage of a fetus. ${ }^{1}$ The maternal pelvis morphological feature is important predictive factor of vaginal delivery.

The female pelvis was classified by Caldwell-Moloy based on shape in 1933. This classification contain four main pelvic types; gynaecoid (50\%), android (25\%), anthropoid (20\%), and platy pelloid $(0.5 \%)$ (Fig. 1$).^{2}$ The prevalence of pelvic varieties is different according to sex and races. ${ }^{3}$

For successful vaginal delivery proper shape and size of the pelvis is necessary. The contracted pelvis leads to disturbances like premature rupture of the membrane, ineffective contractions, abnormal conditions of the fetus, labor dystocia, and finally increasing in rate of cesarean section. ${ }^{3}$ The elective cesarean in these cases could improve the result of delivery. Although cesarean delivery rate is increased in last decades..$^{5-8}$ The result of recent studies showed that rate of cesarean is raised. ${ }^{9-12}$

In normal female pelvis, a longer diameter of the inlet (the transverse diameter) and a longer diameter of the midpelvis (the anteroposterior diameter) are placed perpendicularly. Therefore, a fetal head rotates from a transverse position in the pelvic inlet to a sagittal position in the midpelvis. Narrowing of the pelvic cavity in the midpelvis prevent this rotation. Klemt et al. demonstrated that narrow midpelvis and inadequate proportion of the pelvic inlet causes the emergency cesarean section. ${ }^{13}$ The pelvic inlet and midpelvis are important factors to the anthropological analysis of the female pelvic typology. ${ }^{14}$

Nowadays evaluation of pelvic types based on clinical examination and imaging techniques including CT, MRI, radiography and US are available. ${ }^{14,15}$ The previous studies have shown that pelvimetry with $\mathrm{CT}$ is convenient and

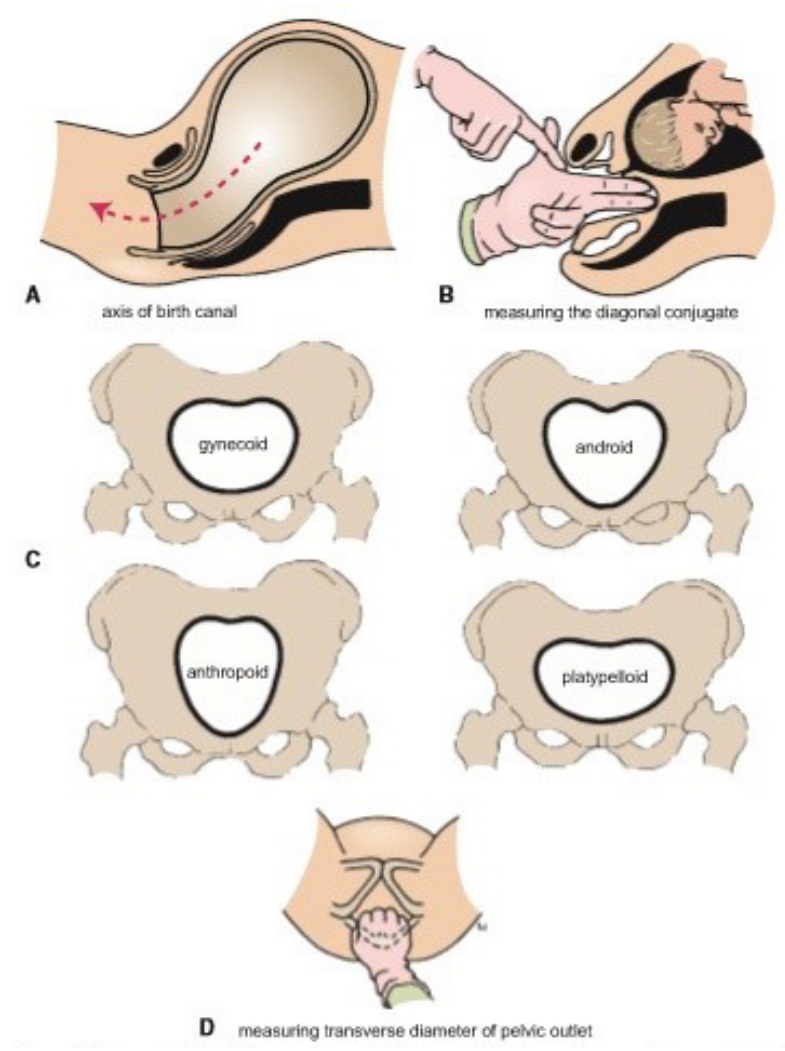

Fig. 1 Different types of pelvic inlets according to classification of Caldwell-Moloy. ${ }^{4}$

accurate technique. ${ }^{16}$ The assessment of pelvic types in addition to another anatomy parameters has clinical importance in predicting the procedures of pregnancy and childbirth in 
reproductive-aged women. In the present work, results of pelvimetry of 157 non-pregnant reproductive-aged women admitted Hazrat-e Rasool university hospital in Tehran evaluated.

\section{Materials and Methods}

\section{Study Design and Participants}

This research was a retrospective study. The clinical records and reports of abdominal CT scans of women referred to radiology department of Hazrat-e Rasool Akram university hospital, Iran University of Medical Sciences, Tehran, Iran during March 2015 to March 2018 were recruited. Subjects according to inclusion and exclusion criteria were eligible. One hundred and fifty-five non-pregnant reproductive-aged women were included and pelvic fractures, lumbosacral spondylolisthesis, pelvic bone tumor, or anomalies and diseases or trauma affected the bony pelvic structure were excluded. In this work, CT examination was conducted using CT scanner (Siemens Medical Systems Inc., Madison, WI, USA). ${ }^{17}$

The obstetrical importance of anteroposterior diameter (diagonal conjugate) is from the tip of the sacral promontory to the lower border of the symphysis pubis (Fig. 2). The true conjugate was determined by measuring the distance from the tip of the sacral promontory to the upper border of the symphysis pubis. The obstetric conjugate is the shortest distance between the tip of the sacral promontory and the most bulging point on the back of symphysis pubis that was measured on the sagittal radiograph. The transverse diameter of the pelvic inlet is the distance between the farthest two points on the iliopectineal lines that were measured on the anteroposterior radiograph. The interspinous diameter of the midpelvis is the distance between the ischial spines that were measured on the axial radiograph. The anteroposterior diameter of the pelvic outlet is determined by measuring the distance from the lower border of the symphysis pubis to the sacrococcygeal joint that was measured on the sagittal radiograph (Fig. 2). The intertuberous diameter of the outlet is the distance between the inner borders of ischial tuberosities that is measured on the anteroposterior radiograph. ${ }^{18}$ The pelvimetry measurements were assessed by the radiographs and using electronic calipers with an internal scale.

\section{Ethics Statement}

This study was performed in accordance with the ethical rules of Helsinki Declaration. All the procedures used in this research was approved by the Ethics Committee of Iran University of Medical Sciences (NO.IR.IUMS.REC.1397.234).

\section{Statistical Analysis}

The obtained data and analytical measurements were recorded and compared. Analysis of collected maternal data including age, gravidity, parity, weight, and height were used as covariates to understand the relationship between pelvic types and kind of delivery. In this study. categorical variables were presented as number (percent) and continuous variables as mean \pm SD with min-max or percentage as appropriate. Statistical analyses were performed using commercially available software (IBM SPSS Statistics version 22; IBM, Chicago, IL, USA). Comparison and correlation of variables were conducted with and correlation tests and a $\mathrm{p}<0.05$ was considered statistically significant.
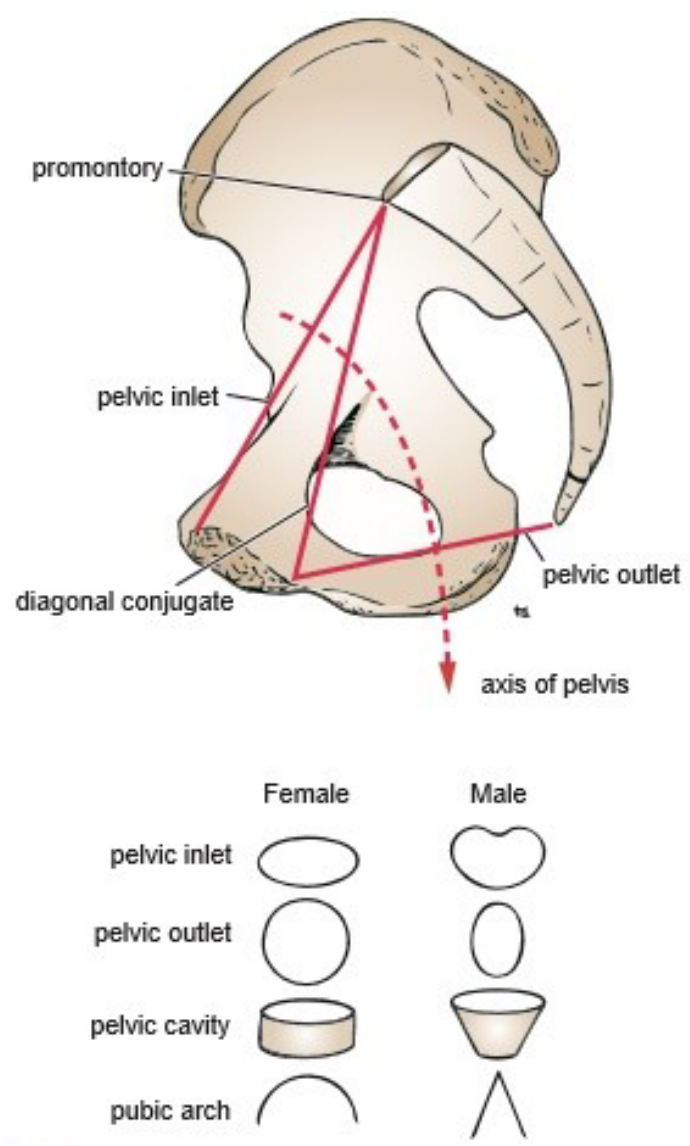

Fig. 2 Pelvic inlet, outlet and diagonal conjugate according to Snell clinical anatomy. ${ }^{4}$

\section{Results}

Pelvic anatomical measurements of 157 subjects were analyzed in the present study. Among the studied subjects, age ranged from 16 to 60 years, height ranged from 150 to $180 \mathrm{~cm}$, and population weight ranged from 43 to $98 \mathrm{~kg}$. Out of these, $102(65.0 \%)$ had at least one normal vaginal delivery (NVD group), and 55 individuals (35.0\%) had undergone at least one cesarean section (C-Sec group). The indication of cesarean delivery was cephalopelvic disproportion. The demographic and anatomical characteristics of the investigated women are summarized in Table 1 . Subjects with cesarean section had a lower height than those with NVD (Mann-Whitney $U$-test, $P$ $=0.035$ ). Besides, the C-Section group of mothers had shorter obstetrical conjugate and interspinous diameter than those in the NVD group $(P<0.05$ for both parameters) (Table 1$)$. No significant difference was observed between two arms of the study in terms of age, weight, parity, true conjugate, diagonal conjugate, transverse diameter, posterior sagittal diameter, intertuberous diameter, and anteroposterior diameter (Table 1).

The frequency distribution of different types of pelvis has been presented in Table 2. The chi square findings unveiled that the relative frequency of android pelvis were statistically higher in $\mathrm{C}$-Sec group $(P=0.036)$. The percentages of other types of pelvis were comparable between the two groups of females (Table 2).

The obtained results demonstrated that scatter plots of pelvic anatomical measurements was stratified according to 


\begin{tabular}{|c|c|c|c|c|}
\hline Demographic/anatomic characteristic & Total $(n=157)$ & $\operatorname{NVD}(n=102)$ & C-Sec $(n=55)$ & $P$-value* \\
\hline Age [yr], median (IQR) & $35(30-39)$ & $35(29-37)$ & $35(31-40)$ & 0.725 \\
\hline Height [cm], median (IQR) & $165(160-169)$ & $167(164-170)$ & $161(157-164)$ & 0.035 \\
\hline Weight [kg], median (IQR) & $70(63-78)$ & $71(64-78)$ & $70(62-77)$ & 0.913 \\
\hline No. of delivery, median (range) & $1(1-8)$ & $1(1-8)$ & $1(1-3)$ & 0.739 \\
\hline True conjugate [mm], median (IQR) & $122.0(114.5-130.0)$ & $123.7(113.0-132.2)$ & $120.1(111.9-131.5)$ & 0.335 \\
\hline Obstetrical conjugate [mm], median (IQR) & $118.2(113.4-125.4)$ & $123.3(115.7-127.1)$ & $113.9(108.0-120.1)$ & 0.013 \\
\hline Diagonal conjugate [mm], median (IQR) & $123.9(114.1-129.5)$ & $126.5(117.3-134.3)$ & $125.5(112.1-131.3)$ & 0.136 \\
\hline Transverse diameter [mm], median (IQR) & $220.3(210.3-230.7)$ & $223.4(212.1-233.1)$ & $219.1(210.0-228.2)$ & 0.259 \\
\hline Posterior sagittal diameter [mm], median (IQR) & $35.2(28.0-39.5)$ & $36.9(29.1-41.2)$ & $35.8(28.9-38.1)$ & 0.417 \\
\hline Interspinous diameter [mm], median (IQR) & $105.0(99.3-110.5)$ & $109.4(104.2-114.1)$ & $101.0(95.4-107.0)$ & 0.029 \\
\hline Intertuberous diameter [mm], median (IQR) & $107.4(101.3-112.5)$ & $109.8(104.4-115.3)$ & $107.2(101.5-111.1)$ & 0.281 \\
\hline Anteroposterior diameter [mm], median (IQR) & $113.3(106.5-117.4)$ & $113.9(105.8-119.0)$ & $111.8(105.5-118.2)$ & 0.363 \\
\hline
\end{tabular}

*Computed by Mann-Whitney U-test

Abbreviation: C-sec, Cesarean section, IQR, interquartile range (25th-75th percentile); NVD, Normal vaginal delivery

\begin{tabular}{|c|c|c|c|c|}
\hline Type of pelvis & Total $(n=157)$ & $\operatorname{NVD}(n=102)$ & $C-\operatorname{Sec}(n=55)$ & $P$-value* \\
\hline Gynaecoid pelvis (Round), n (\%) & $91(58.0)$ & $62(60.8)$ & $29(52.7)$ & 0.716 \\
\hline Android pelvis (Transverse oval), $n$ (\%) & $14(8.9)$ & $5(4.9)$ & $9(16.4)$ & 0.036 \\
\hline Anthropoid pelvis (Long oval), $\mathrm{n}(\%)$ & $14(8.9)$ & $8(7.8)$ & $6(10.9)$ & 0.564 \\
\hline Platypelloid pelvis (Flat), $n$ (\%) & $38(24.2)$ & $27(26.5)$ & $11(20.0)$ & 0.437 \\
\hline
\end{tabular}

*Computed by Fisher's exact test

the median age of the population. Statistical analysis revealed that the median values of true conjugate (Fig. 3A), obstetrical conjugate (Fig. 3B), diagonal conjugate (Fig. 3C), transverse diameter (Fig. 3D), posterior sagittal diameter (Fig. 3E), and interspinous diameter (Fig. 3F) were significantly lower in women aged $<35$ years than women aged $>35$ years in C-Sec group $(P<0.05)$. Although no significant differences were shown between women more or less than 35 years of age who had undergone spontaneous vaginal delivery (NVD group) (Fig. 3A-H).

\section{Discussion}

In this study, all 157 subjects had a true conjugate (122.0 \pm $9.0 \mathrm{~mm}$ ) and a diagonal conjugate $(123.9 \pm 9.5 \mathrm{~mm})$. The obstetrical conjugate was $123.3 \mathrm{~mm}$ in NVD and $113.9 \mathrm{~mm}$ in C-sec. It was slightly longer than the delivery group. This difference in diameter of conjugates is assumed to originate from the measurements made in younger women who have a cesarean section and they have not experienced vaginal delivery.

The cesarean-section is also called cesarean delivery. However, the C-section has many risks such as wound infection, blood loss, injury to an organ just like the gastrointestinal tract or bladder, adverse reactions, although the cesarean-section has been grown in Iran. ${ }^{19}$

Regarding to evidences based on the anatomy and anthropology sciences, the pelvimetry technique has an important role in determination of the bony pelvis diameters and estimating the pelvic diameters. ${ }^{1}$

In this study, results of pelvimetry using CT in study population and its association with increased cesarean section rate were evaluated. In this study, the height of mothers did not match with type of delivery and pelvis dimensions. Furthermore, no relationship was found between pelvis type and body mass index.

Previous studies have shown that maternal height is related to the risk of cesarean section.

Cesarean section rises with shortness of maternal height. ${ }^{20}$ The data of our study showed that the gynaecoid pelvis (58\%), platypelloid (24.2\%), anthropoid (8.9\%), and android (8.9\%). The size and shape of the pelvic canal vary in women, and the gynecoid pelvis is usually the most appropriate type of pelvis for vaginal delivery. However, we know that Android and platypelloid are not suitable for childbirth. A previous study showed that mothers with gynecoid pelvis had better outcomes $(71.6 \%-47.8 \%){ }^{21}$

In this research, we evaluated the pelvic diameter in women $\leq 35$ - and $\geq 35$ years old. Our result proved that the inlet and midpelvis diameters of $<35$ years old group are significantly decreased in compared to the $>35$ years old group.

Lin et al indicated that there was a link between increasing maternal age and tendency to cesarean section. ${ }^{22}$ But in the present study, the rate of the cesarean section has been more in $<35$ years old. 
A

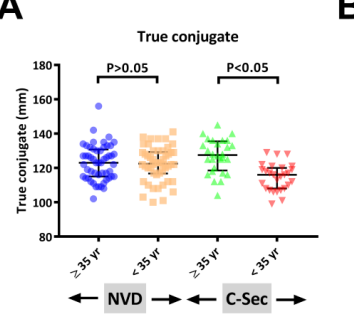

C

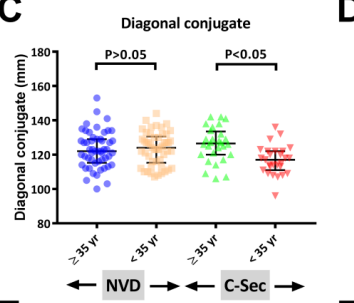

E

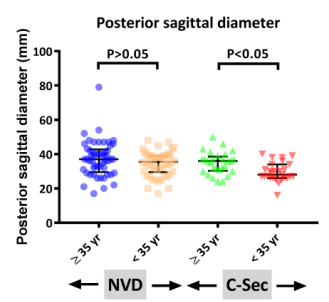

G

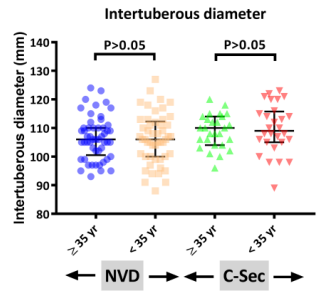

B

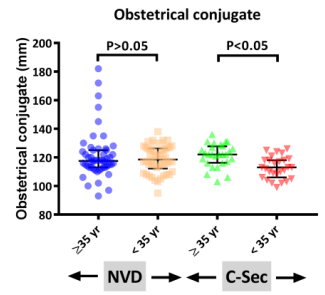

D

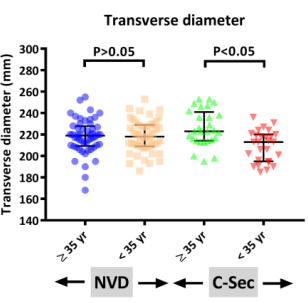

$F$

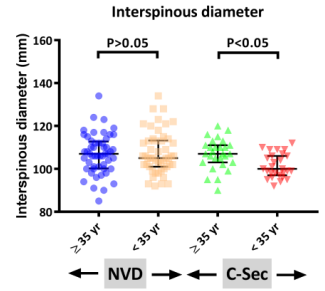

H

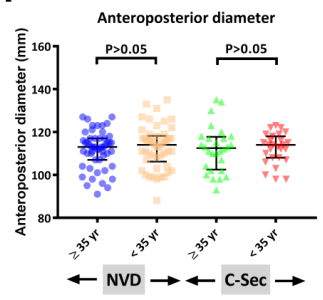

Fig. 3 Scatter plot of (A) true conjugate, (B) obstetrical conjugate, (C) diagonal conjugate, (D) transverse diameter, (E) posterior sagittal diameter, (F) interspinous diameter, (G) intertuberous diameter, and $(\mathrm{H})$ anteroposterior diameter in subjects with $\geq 35$ and $<35$ years of age who had undergone either NVD (normal vaginal delivery) or C-Sec (cesarean section).

\section{Conclusion}

Regarding the results of this study, shortening of diameter of the inlet and midpelvis causes the increase in rate of cesarean section and it has been more in females $<35$ years old. This study need to consider more variables such as economic status and psychological conditions.

\section{Acknowledgements}

This research was funded by Iran University of Medical Sciences, fund grant IR.IUMS.REC.1397.234. The authors would like to thank our colleagues at Hazrat-e Rasool university hospital.

\section{Conflict of interest}

The authors declare no conflict of interest.

\section{References}

1. Aubry S, Padoin P, Petegnief Y, Vidal C, Riethmuller D, Delabrousse E. Can three-dimensional pelvimetry using low-dose stereoradiography replace low-dose CT pelvimetry? Diagn Intervent Imaging. 2018;99(9):569-76.

2. Caldwell W, Moloy HC. Anatomical variations in the female pelvis and their effect in labor with a suggested classification. Am J Obstet Gynecol. 1933;26(4):479-505

3. Williams Obstetrics. 23th Edition.Translation: Valadan M RS, Fathollahi A. Tehran: Arjmand Medical Publisher; 2010. volume 2. p. 141-142.

4. s.snell R. clinical_anatomy_by_regions 2012 .

5. Bregar AT. Indications for caesarean delivery between 1955 and 2005 Indicationen fuer Kaiserschnitt geburth zwieschen 1955 und 2005.

6. Eskew JP, Saywell JR, Zollinger T, Erner B, Oser T. Trends in the frequency of cesarean delivery. A 21-year experience, 1970-1990. J Reprod Med. 1994;39(10):809-17.

7. Krychowska A, Kosińska K, Karwan-Płońska A. Comparison of indications for cesarean section in 1985-86 and 2000-01. Analysis of changes. Ginekologia polska. 2004;75(12):926-31.

8. Tampakoudis P, Assimakopoulos E, Grimbizis G, Zafrakas M, Tampakoudis $G$, Mantalenakis S, et al. Cesarean section rates and indications in Greece: data from a 24-year period in a teaching hospital. Clin Exp Obstet Gynecol. 2004;31(4):289-92.

9. Shareferad G, Fathean Z, Terane M, Mahake B. The Survey of pregnant women views about delivery and cesarean according behavioral intention model. Ilam Uni Med Sci J. 2007;15:19-23.

10. Bani S, SEIED RA, SHAMSI GT, Ghojazadeh M, Hasanpoor S. Delivery Agents Preferences Regarding Mode of Delivery for Themslves and Pergnant Women (Obstetrics, Gynecologists, Midwives). 2010

11. Rafiei M, Saei Ghare M, Akbari M, Kiani F, Sayehmiri F, Sayehmiri K, et al. Prevalence, causes, and complications of cesarean delivery in Iran: A systematic review and meta-analysis. Int J Reprod Biomed (Yazd, Iran). 2018;16(4):221-34.

12. Azami-Aghdash S, Ghojazadeh M, Dehdilani N, Mohammadi M, Asl Amin Abad R. Prevalence and causes of cesarean section in Iran: Systematic review and meta-analysis. Iran J Public Health. 2014;43(5):545-55.

13. Klemt A-S, Schulze S, Brüggmann D, Louwen F. MRI-based pelvimetric measurements as predictors for a successful vaginal breech delivery in the Frankfurt Breech at term cohort (FRABAT). Eur Jof Obstet Gynecol Reprod Biol. 2019;232:10-7.

14. Perlman S, Raviv-Zilka L, Levinsky D, Gidron A, Achiron R, Gilboa Y, et al. The birth canal: correlation between the pubic arch angle, the interspinous diameter, and the obstetrical conjugate: a computed tomography biometric study in reproductive age women. J Matern-Fetal Neonat Med. 2018:1-11.

15. Zaretsky MV AJ, Menintire DD, Hatab MR, Leveno KJ. Magnetic resonance imagining pelvimetry and the prediction of labor dystocia. Obstet Gynecol. 2005;106(5):919-926.

16. Kolesova O, Kolesovs A, Vetra J. Age-related trends of lesser pelvic architecture in females and males: A computed tomography pelvimetry study. Anat Cell Biol. 2017;50(4):265-74.

17. Federle MP, Cohen HA, Rosenwein MF, Brant-Zawadzki MN, Cann CE. Pelvimetry by digital radiography: A low-dose examination. Radiology. 1982;143(3):733-5.

18. Morris CW, Heggie JC, Acton CM. Computed tomography pelvimetry: Accuracy and radiation dose compared with conventional pelvimetry. Austr Radiol. 1993;37(2):186-91.

19. Ham SJ, Koops HS, Veth RP, van Horn JR, Eisma WH, Hoekstra HJ. External and internal hemipelvectomy for sarcomas of the pelvic girdle: consequences of limb-salvage treatment. Eur J Surg Oncol (EJSO). 1997;23(6):540-6.

20. Liselele HB, Boulvain M, Tshibangu KC, Meuris S. Maternal height and external pelvimetry to predict cephalopelvic disproportion in nulliparous African women: A cohort study. BJOG Int J Obstet Gynaecol. 2000;107(8):947-52.

21. Salk I, Cetin M, Salk S, Cetin A. Determining the incidence of gynecoid pelvis using three-dimensional computed tomography in nonpregnant multiparous women. Med Princip Pract. 2016;25(1):40-8.

22. Lin HC, Sheen TC, Tang CH, Kao S. Association between maternal age and the likelihood of a cesarean section: a population-based multivariate logistic regression analysis. Acta obstet gynecol Scand. 2004;83(12):1178-83.

This work is licensed under a Creative Commons Attribution-NonCommercial 3.0 Unported License which allows users to read, copy, distribute and make derivative works for non-commercial purposes from the material, as long as the author of the original work is cited properly. 